Cahiers de recherches médiévales

15 | 2008

La Tentation du parodique dans la littérature médiévale

\title{
La parodie en situation
}

Approches du texte festif de la fin du Moyen Âge

Jelle Koopmans

\section{CpenEdition}

Journals

Édition électronique

URL : https://journals.openedition.org/crm/5603

DOI : $10.4000 / \mathrm{crm} .5603$

ISSN : 1955-2424

Éditeur

Honoré Champion

Édition imprimée

Date de publication : 20 juin 2008

Pagination : 87-98

ISSN : $1272-9752$

Référence électronique

Jelle Koopmans, «La parodie en situation », Cahiers de recherches médiévales [En ligne], 15 | 2008, mis en ligne le 20 juin 2011, consulté le 15 décembre 2022. URL : http://journals.openedition.org/crm/ 5603 ; DOI : https://doi.org/10.4000/crm.5603 


\title{
酷RM
}

\section{La parodie en situation : approches du texte festif de la fin du Moyen Âge}

\begin{abstract}
This article argues that for many late medieval texts, parody defines itself by the inscription in a larger context of festivities. Often parody is not determined by inversion of a given textual structure, but on the contrary as a logical component of a topsy-turvy world.

Résumé :Pour bien des textes de la fin du Moyen Âge, la parodie se définit par l'inscription dans le cadre de festivités. Souvent, la parodie n'est pas déterminée par l'inversion d'une structure textuelle donnée, mais plutôt comme composante logique d'un monde à l'envers.
\end{abstract}

Même si, selon certains érudits, le Moyen Âge a ignoré le concept de la parodie («le concept est d'autant plus délicat que le Moyen Âge l'a ignoré »1), la parodie, au Moyen Âge, semble avoir été partout (ce qui n'est point trop difficile pour une si longue période). Et on est certes en droit de se poser la question de savoir si c'est bien une approche purement conceptuelle qui va bien nous aider à saisir exactement ce que la parodie médiévale a bien pu être. On confrontera avec fruit la remarque de Jean-Claude Mühlethaler, selon qui le Versus de Unibove constitue l'« un des rares textes latins à caractère ludique qui nous ont été conservés " ${ }^{2}$, à celle de Martha Bayless, selon qui rien ne montre mieux la popularité de la parodie médiévale que le nombre de manuscrits conservés ${ }^{3}$. Autant dire que l'on retombe facilement sur des questions de définition ou de conceptualisation qui ne sont pas forcément les questions les plus intéressantes ni les plus fructueuses à traiter ici. Il est même tentant de suggérer que l'on a cherché la parodie où elle n'était pas sans trop s'embarrasser de la trouver où il fallait bien la chercher. Permettons-nous tout de même de signaler que des genres littéraires comme la sote chanson, comme le sermon joyeux, comme le testament ou le mandement joyeux, comme la pronostication joyeuse qui ont tout pour nous convaincre de la présence de la pensée, de l'intention, du genre parodique au Moyen Âge montrent qu'une forme de conceptualisation de la parodie s'est bien produite, ne fût-ce qu'implicitement. Les multiples commentaires qu'a pu susciter un texte comme Aucassin et Nicolette, texte en effet plutôt curieux, chantefable littéralement unique en son genre, sont symptomatiques : là où une multiplicité d'éventualités sur le plan de l'intertexte se dessine, le genre «chantefable», dans ses possibilités performatives (Or dient, or se cante) a dû déterminer une bonne sinon une majeure partie de son potentiel parodique. Autant dire que là aussi, probablement, la parodie

\footnotetext{
1 J.-Cl. Mühlethaler, «Préface: À la recherche de la parodie médiévale », Formes de la critique: Parodie et satire dans la France et l'Italie médiévales, Études publiées par J.-Cl. Mühlethaler avec la collaboration d'A. Corbellari et de B. Wallen, Paris, Champion, 2003, p. 7.

${ }^{2}$ Op. cit., p. 11 .

${ }^{3}$ M. Bayless, The Medieval Parody, Ann Arbor, University of Michigan Press, 1996.
}

Cahiers de Recherches Médiévales, 15, 2008 
a dû résider au niveau de la situation - mais, faute d'informations concrètes, on en est réduit à de pures spéculations. Autant dire que ce texte, chantefable unique en son genre- et seul représentant du genre- reste isolé: contextualisé par les intertextes établis à partir d'un canon constitué bien après le Moyen Âge, mais historiquement un orphelin. Ce qui, vers la fin du Moyen Âge, facilite bien la tâche du philologue, c'est une documentation bien plus riche et bien plus précise, encore que toujours bien lacunaire, au sujet des situations de jeu, de performance, de présentation ou de représentation des textes.

Ces dernières remarques sont là puisqu'elles tendent à montrer que ni la seule constatation d'intertextes, souvent formulée a posteriori, ni la seule conceptualisation de la parodie au niveau générique ne suffisent pour épuiser le sujet de la parodie médiévale, car on risquerait de se laisser tenter par une approche par trop « littéraire ». Le texte médiéval est souvent, de par sa nature performative, lié à des circonstances concrètes plutôt qu'à un «canon littéraire» et ce n'est pas nécessairement une pensée générique qui préside à la création de textes parodiques. L'enjeu, ici, sera de montrer par une suite d'exemples concrets comment cette performativité ou, si l'on préfère, cette mise en situation du texte parodique arrive à mieux faire comprendre le fonctionnement de l'espace littéraire de l'inversion. C'est que, en effet, là où l'inversion littéraire se fait jour, il est le plus souvent question de circonstances concrètes qui déterminent, voire nécessitent une telle inversion. L'espace de la performativité parodique a son importance; c'est bien à partir d'une contextualisation situationnelle que la parodie prend, le plus souvent, son plein sens. De quelque manière qu'on veuille définir la parodie, force nous est de constater que l'inversion, et spécialement l'inversion festive, ont une place centrale : la parodie, au lieu d'être simplement textuelle, paraît bien se définir à partir d'un lieu de l'inversion. Ainsi, le sermon joyeux ne se définit pas nécessairement à partir du sermon sérieux par un rapport d'inversion; au contraire, une telle homélie parodique peut bien être, au sein d'un monde festif, d'un mundus inversus parodique, un élément qui retrouve sa place naturelle : là où le monde «sérieux » exige un sermon, le monde festif nécessite un sermon joyeux - ce qui suppose, tout de même, des rapports légèrement différents. L'ouvrage de Bakhtine sur Rabelais, certes y est pour quelque chose, même si l'on ne soulignera jamais assez à quel point il propose des interprétations en somme quelque peu gratuites à partir d'un matériel documentaire trop restreint, mais n'oublions pas que les études de Lehmann et de Bayless, bien mieux documentés, vont dans le même sens ${ }^{4}$. Constatons en outre que, par le biais d'une méthode qui marie harmonieusement l'histoire de la littérature à l'anthropologie ou "folklore» français, Jacques Merceron arrive à dégager un matériel riche, une longue durée tout à fait étonnante au sujet des saints facétieux et burlesques; de la sorte, il faut le dire, le concept de la parodie médiévale est problématisé dans sa continuité, remis en question dans sa spécificité, illustré dans sa richesse 5 .

Avant, toutefois, d'en arriver à de telles questions générales, il faut bien faire ses gammes. En l'occurrence cela veut dire aussi, et surtout, un repli sur les textes concrets. Un premier point à prendre en considération, c'est ce que j'ai pu appeler

\footnotetext{
${ }^{4}$ M. Bayless, op. cit. ; P. Lehmann, Die Parodie im Mittelalter, Stuttgart, 1963.

${ }^{5}$ J. Merceron, Dictionnaire des saints imaginaires et facétieux, Paris, Seuil, 2002.
} 
l'indication de la parodie. Dans mon édition du corpus des sermons joyeux français des $X^{\mathrm{e}}$ et $\mathrm{XVI}^{\mathrm{e}}$ siècles, j'ai mis l'accent sur ce que j'ai appelé «l'indication de la parodie ", c'est-à-dire sur les manières qu'utilise le texte de s'afficher (souvent d'emblée) comme parodique ${ }^{6}$. Tel sermon joyeux annonce son caractère parodique dans le titre, telle farce s'annonce «fort joyeuse», telle pronostication joyeuse annonce d'emblée qu'ici, finalement, on trouvera autre chose que dans les textes sérieux, que tous les autres sont de charlatans mais que maintenant, on entendra une prédiction authentique et véritable. Bien évidemment, le critère a un côté faible, car bien des textes sérieux annoncent dès le début que tous les prédécesseurs ont oublié de donner le meilleur de la matière : c'est un poncif qui va des branches du Roman de Renart aux pronostications sérieuses, c'est un lieu commun dans bien des sermons, sérieux ou joyeux, du XII ${ }^{\mathrm{e}}$ siècle jusqu'à l'humanisme. En même temps, c'est, dira-t-on, une ouverture conflictuelle d'un texte qui a tantôt rapport à un prétexte, à un intertexte ou aux conventions d'un genre, mais qui tantôt porte uniquement sur les qualités du personnage, d'un certain concept de rôle. Dans l'Erberie en prose, par exemple, l'acteur commence par «Bele gent, je ne sui pas de ces povres prescheurs, ne de ces povres herbiers... $\gg^{7}$. Les farces, sermons et pronostications sont explicites au sujet de leurs intentions : ils sont «joyeux», «nouveaux » et " pour rire ». " A celle fin que puissons dire / Chose de quoy nous puissions rire» nous précise bien le Sermon joyeux à tous les fous ${ }^{8}$; Sermon joyeux pour rire s'intitule bien, dans le manuscrit La Vallière, un sermon au sujet du mariage ${ }^{9}$; Sermon fort joyeux pour faire rire et est de saint Raisin est bien le titre, dans le Recueil Trepperel du sermon bachique au sujet du martyr de la vigne ${ }^{10}$.

Si la parodie peut se définir comme l'inversion du signifié dans une structure signifiante fixe ${ }^{11}$, ou, selon l'heureuse formulation de Paul Zumthor, comme la figure de l'ironie «en vertu de laquelle la fonction d'un texte se trouve remplie par un autre, de sorte qu'un contenu et une expression en principe indissociables sont détachés $\rangle^{12}$, la parodie a tendance à $s$ 'afficher comme telle. Songeons à la fleur inverse des troubadours, songeons aux sottes chansons, au genre de la sote amoureuse. Au printemps de la canso répond un hiver, aux sujets élevés des mystères répondent des interludes comiques (diableries, scènes avec des vilains). Même si l'on ne dira plus, avec Bédier (au sujet des fabliaux), qu'à la poésie des châteaux s'oppose une poésie des carrefours ou de la place publique, on peut bien constater qu'un jeu conscient entre ce qui est perçu comme une «haute» culture, une culture noble, et une " basse culture », englobant des éléments avec une certaine précision anatomique, n'est pas trop éloigné de la littérarité médiévale.

Sur un plan plus proprement historisant toutefois, on constatera le caractère nécessairement implicite de la parodie dans bien des cas, pour la bonne et simple raison que la situation performantielle a dû déterminer le caractère parodique qui,

\footnotetext{
${ }^{6}$ J. Koopmans, Recueil de sermons joyeux, Genève, Droz, 1988, p. 49.

${ }^{7}$ E. Faral et J. Bastin, Euvres complètes de Rutebeuf, Paris, Picard, 1977 t. II, p. 276.

${ }^{8}$ Recueil de sermons joyeux, p. 264.

${ }^{9}$ Ibid., p. 546-552.

${ }^{10}$ Ibid., p. 533-542.

${ }^{11}$ W. Karrer, Parodie, Travestie, Pastiche, Munich, UTB, 1977, p. 81 et suivantes.

${ }^{12}$ P. Zumthor, Essai de poétique médiévale, Paris, Seuil, 1972, p. 104.
} 
pour le lecteur moderne du texte qui constitue la retombée de cette situation, n'est plus toujours évidente. Un premier exemple pourrait être la Farce des trois nouveaux martyrs (Cohen, $\mathrm{XL}^{13}$ ). Les trois martyrs, ici, sont le martyr marié, le martyr en procès et le martyr en ménage, qui cherchent à être couronnés comme de véritables martyrs du calendrier chrétien, ce qui constitue un premier pas dans la démarche parodique. Heureusement, il y a le personnage allégorique appelé La Fin qui réalise sur scène le proverbe La fin couronne l'œuvre; en effet, ce personnage allégorique couronnera, à la fin, nos trois martyrs. En cela, on peut parler d'une réalisation scénique d'une locution ou d'un proverbe. Toujours est-il que le texte de la farce se structure d'après la «litanie mineure» ou les Rogations - qui est à distinguer de la Grande litanie de la Saint-Marc (le 25 avril). La structure textuelle est nette: Christe, audi nos... (v. 1-27); Ora pro nobis... (v. 48-53) ; Libera nos... (v. 54-65); Te rogamus, audi nos... (v. 66-774). À propos des Rogations, Van Gennep spécifie «le rite oral se complique aussi de litanies qui semblaient restées immuables au cours des siècles et qui comportent le répons collectif Te rogamus audi nos $\gg{ }^{14}$. D'autres emplois de la litanie parodique se laissent facilement deviner : A dialectica libera nos, domine, chantaient les étudiants ; A Litaniis praedicatorum libera nos, domine, chantaient les hétérodoxes; A FELICE Sicilia, libera nos domine, A Corsica et Sardinia, libera nos, domine, tel est finalement le chant d'un évêque, et plus proche du contexte des procès : A cibo bis cocto / A servo nimis docto, / A procuratore et advocato / Ab amico reconliato, / Ab auditore camere ${ }^{15}$. Le rhétoriqueur Jean Molinet utilisera, parodiera la même structure dans sa Letania Minor $^{16}$. La sottie du Pèlerinage de Mariage ${ }^{17}$ contient également une «litanie mineure » chantée par l'ensemble des acteurs à la fin de la sottie, avec une série impressionnante de pseudo-saints. Autant dire que le cas est loin d'être isolé. Le texte de notre farce est calqué directement sur la structure des Rogations et c'est dans cette intention parodique que nos pauvres martyrs adressent leurs Ora pro nobis à des saints comme "saint Debet-Testu », " saint Cogne-Festu », " saint Jouan-Cocu », mais aussi à des noms de saints plus truqués comme " saint Porteurde-douleances-et-d'appeaulx », « saint Ouvrier-de-faire-de-tous-boys-coypeaux » et « saint Souffrant-qu'on larde-a-ta-femme-ses-peaux ». Si l'on passe sur le caractère ouvertement parodique - et parfois même doublement parodique ${ }^{18}-$ des saints en question, il faut noter qu'ici donc, l'explicité du texte permet facilement de reconstruire une situation communicative et de voir, par là, l'enjeu parodique apparent du texte. Ce qui n'est pas, malheureusement, toujours le cas. Dans ce cas concret, la farce se présente implicitement comme une parodie des litanies mineures ce qui - en vertu d'un contexte performantiel - a dû être tout à fait clair pour le spectateur médiéval, ce qui, probablement, a même été déterminé par la situation

\footnotetext{
${ }^{13} \mathrm{G}$. Cohen, Recueil de farces françaises inédites $d u X V^{e}$ siècle, Cambridge (Mass.), 1949.

${ }^{14}$ A. Van Gennep, Le Folklore français, Paris, Laffont, 1998-1999, p. 1359, 1364.

${ }^{15}$ M. Bayless, op. cit., p. 120.

${ }^{16}$ N. Dupire, Jean Molinet, Les Faictz et Dictz, Paris, SATF, 1936-1939, p. 548-554.

${ }^{17}$ E. Picot, Recueil général de sotties, Paris, SATF, 1902-1912, t. III, p. 269-300.

${ }^{18}$ Le saint burlesque saint Souffrant, du fameux «baiser saint Soufrant» (J. Merceron, op. cit., p. 379-381), à nouveau replacé dans une structure syntaxique souffrant qu'on larde a ta femme les peaux (« cocu complaisant »).
} 
concrète de jeu, mais ce qui n'est plus apparent pour un lecteur moderne ni, peutêtre, pour un lecteur du XVI ${ }^{\mathrm{e}}$ siècle confronté à un recueil factice de 53 farces. N'oublions pas, toutefois, que l'exemplaire relié dans le recueil Cohen n'est qu'un exemplaire, fortuitement conservé, d'un tirage plus important, destiné éventuellement à un autre public. Même si nous ignorons tout au sujet de la situation concrète de jeu - des hypothèses pourront être formulées à ce sujet - la structure de cette farce montre clairement que, pour cette occurrence, la farce est contextuelle ou elle n'est pas. La situation de jeu prime sur l'éventuelle question générique : c'est bien d'une farce pour des litanies qu'il est question ici.

Un problème d'interprétation analogue est défini par l'intentionnalité du texte. C'est que, pour toute interprétation valide d'un texte complexe, il ne suffit pas d'isoler certains éléments, mais il faut encore les relier à l'ensemble, osera-t-on dire une structure. C'est ce qui a fait la force des analyses proposées par Robert Darnton pour le grand massacre de chats au XVIII ${ }^{\mathrm{e}}$ siècle parisien, c'est ce qui fait la valeur de l'analyse du Carnaval de Romans (1580) proposée par Leroy Ladurie. La leçon, toutefois, demande à être élargie. C'est que, dans certains mystères par exemple, l'on rencontre bien des scènes qui peuvent être qualifiées de parodiques, comme des scènes de torture et de martyre, comme des diableries. Le sont-elles ? En fait, la réponse à cette question devrait être plutôt complexe. Ce qui a une fonction parodique à l'intérieur d'un texte ne l'a pas nécessairement par rapport à l'extérieur, à l'intertexte, à la situation - en même temps, ce qui est parodique par rapport à l'extérieur ne revêt pas nécessairement une fonction parodique à l'intérieur du texte. Tantôt un passage, ou une scène, peuvent bien être vus comme parodiques à partir de notre connaissance d'intertextes divers, ou de situations concrètes; reste à se demander si le public de l'époque partageait notre bagage culturel et était à même de saisir le lien. Dans certains cas, comme dans celui du ré-emploi de l'axe CarnavalCarême dans des contextes hagiographiques, la référence ne fait guère de doute. Les bourreaux des mystères avec des noms comme Happelopin, Tailleboudin ou Rifflandouille entrent naturellement dans cette catégorie; étant donné que le monde de Satan, et des Païens, est une cuisine, une cuisine grasse, un Cocagne contraire aux vertus de l'abstinence, les adversaires de la foi sont investis d'un sens rigoureusement contemporain, définissant une axiologie bourgeoise de la fin du Moyen Âge. Reste bien sûr à se poser la question de savoir si de tels personnages peuvent également revêtir une fonction parodique à l'intérieur de la pièce, et là, forcément, l'analyse demande un peu plus de réserves. Bien sûr, l'épistémologie de la ressemblance et la topologie du genre expliquent pour une bonne part pourquoi, dans les mystères hagiographiques, l'opposition entre la Chrétienté et le monde païen doit se doubler d'un axe qui va de la cuisine grasse (mais c'est aussi le Carnaval) à la cuisine maigre (mais c'est aussi le Carême), d'un axe qui va de l'hiver rigoureux au printemps avec son abondance de vivres, qui va de l'ascèse chrétienne à la gula des infidèles. Ce qui joue, dans de tels cas, c'est le ré-emploi de certaines structures empruntées à des rituels d'inversion dans des contextes dramatiques où elles sont appelées uniquement par des «ressemblances». L'opposition entre Païens et Chrétiens, dans les mystères, est souvent redéfinie sur la base d'autres axes bi-polaires, comme Carnaval-Carême, on vient de le voir, comme la ville et la barbarie campagnarde. L'enfer étant une cuisine, le martyre devient une question relevant de la cuisine grasse, donc du Carnaval: dans le 
Mystère de saint Quentin, le bourreau Fourrure veut rôtir les tripes et boyaux des Chrétiens boursouflés; son compère Tempeste veut les tailler par rouelles comme des gros boudins enflés ${ }^{19}$. De telle recodifications carnavalesques ne sont pas toujours innocentes. N'oublions pas qu'un tirant du nom Tailleboudin peut bien renvoyer au capitaine anglais Tallebot, mais aussi au nom proverbial du cuisinier Taillevent. Dans le cas du Dialogue entre MM. de Mallepaye et Baillevent, ce dernier renvoi s'impose même - et de là, on commence à se poser de sérieuses questions autour du sobriquet Taillevent du cuisinier Guillaume de Tirel, auteur du Viandier, mais aussi autour du nom du poète Michault Taillevent, enfin, du personnage que nous connaissons comme poète, mais que les sources contemporaines désignent généralement comme le plus grand farceur de la cour de Bourgogne. Le subtil jeu entre de tels axes est tout à fait clair dans la moralité de la Condamnation de Banquet, où une telle superposition de codes est nettement inscrite dans le texte même ${ }^{20}$. C'est que cette pièce spectaculaire, où les excès de bouche sont condamnés, copie la structure des multiples martyres dans les mystères, mais ici, la victime sera Banquet (représentant la cuisine grasse) et les bourreaux (Pillule, Diète, Remède) représenteront la cuisine maigre. En même temps, la condamnation rituelle d'un représentant de la cuisine grasse rappelle naturellement à l'esprit le rituel de la mort de Carnaval, de Carême-Prenant. En tout cela, n'oublions pas que l'intentionnalité reste le plus grand obstacle à la compréhension de la littérature historique : si seulement on avait une date pour la pièce, une inscription calendaire, bien des obstacles à l'interprétation pourraient rapidement être résolus.

Toutefois, ici comme ailleurs, ce que j'ai appelé dans ma thèse l'« indication de la parodie $»^{21}$, c'est-à-dire l'explicitation de l'intention, vient souvent à notre secours. Wolfgang Karrer a bien montré comment, d'après bien des publications, la parodie serait un fait social, à relier au Carnaval ou plus en général à la fête, avant de conclure que de telles observations restent, pour l'instant, isolées et que des hypothèses générales à ce sujet restent à formuler ${ }^{22}$. Depuis lors, certaines études ont illustré ce point à partir d'analyses ponctuelles de cas précis - songeons notamment au Carnaval de Romans de 1580 (Le Roy Ladurie), au grand massacre de chats au $\mathrm{XVIII}^{\mathrm{e}}$ siècle (Darnton), aux rituels lyonnais du $\mathrm{XVI}^{\mathrm{e}}$ siècle (Davis). Pour rester dans un contexte bakhtinien, Karrer met également l'accent sur le haut et le bas, ou plus généralement, sur une préoccupation avec ce qui est commun, bas ou obscène $^{23}$ - un accent qui se comprend (mais que je ne pourrai faire mien), encore qu'il tende à simplifier les choses à l'extrême. Dans un examen précis du fonctionnement parodique, c'est le fait social, ce sont les modalités de la communication - et par là aussi le contexte situationnel - qui doivent primer sur tout a priori anachronique.

${ }^{19}$ H. Châtelain, Le Mistere de saint Quentin suivi des invencions du corps de saint Quentin par Eusèbe et Eloy, Saint-Quentin, 1907, p. 33.

${ }^{20} \mathrm{~J}$. Koopmans, "Une pièce parodique à trois codes implicites: La Condamnacion de Banquet de Nicolas de la Chesnaye », Fifteenth-Century Studies, 18, 1991, p. 159-174.

${ }^{21} \mathrm{~J}$. Koopmans, Recueil général des sermons joyeux français ( $X V^{e}-X V I^{e}$ siècles), Leiden, thèse, 1987, p. 72-73 ; voir Recueil des sermons joyeux, p. 49.

${ }^{22}$ W. Karrer, op. cit., p. 21.

${ }^{23}$ Ibid., p. 25. 
Prenons, afin d'illustrer l'importance du fonctionnement social du texte parodique ainsi que l'urgence d'étudier la parodie dans son contexte large, situationnel, l'exemple de Caen en 1492. À cette date, pendant le Carnaval, on joue la Farce de Pates-Ouaintes ${ }^{24}$, qui a été conservée dans le Matrologe de l'Université de Caen (Caen, Musée des Beaux-Arts, coll. Mancel, ms.69). Cette pièce a été attribuée à Pierre de Lesnauderie, qui en effet l'a signée dans le manuscrit (voir au sujet de cet auteur l'article d'Estelle Doudet dans ce même volume). Cette pièce (est-ce bien une farce ?) met en scène l'ancienne image de la mère mangée par ses enfants ainsi que de l'agneau mangé par le loup. La combinaison des images est saugrenue, il faut le dire. Toutefois, le contexte nous éclaire sur le fonctionnement de cette pièce et - une fois n'est pas coutume - le manuscrit du Matrologe nous donne le plein d'informations à ce sujet. Qu'est-il arrivé ? L'Université de Caen a été fondée, au début du $X V^{\mathrm{e}}$ siècle, par le roi d'Angleterre, ou, pour être plus précis, par celui qui se considérait - à juste titre d'ailleurs - comme le roi de France (mais qui occupait en même temps la fonction de roi d'Angleterre). À sa fondation, elle a reçu le privilège d'une exemption des taxes et tout au long du $X^{\mathrm{e}}$ siècle (et audelà), jalouse de ses privilèges, l'Université de Caen n'a cessé de les défendre, de les faire défendre par les «conservateurs des privilèges». N'oublions pas que les privilèges universitaires ont été un sujet de controverse, de conflits multiples tout au long des $X^{\mathrm{e}}$ et $\mathrm{XVI}^{\mathrm{e}}$ siècles; vers la fin de la décennie 1490-1500, cette question mène à une cessation des leçons à l'Université de Paris et à l'exil de Jean Standonck. Retournons à notre agneau caennais. Au moment où, vers la fin du $\mathrm{XV}^{\mathrm{e}}$ siècle, le roi de France décide d'imposer la décime aux suppôts de l'Université de Caen, justement pour contrer une invasion imminente du roi d'Angleterre (!), les réactions sont violentes dans le monde universitaire caennais. Des anciens suppôts de l'université se prêtent à la perception de cette décime, le roi de France, avec le soutien du pape, mobilise les évêques, mais les évêques qui doivent défendre les privilèges de l'université s'y opposent. Si l'on me permet une petite parenthèse ici, le cas est tout à fait exemplaire pour la grande crise d'autorité, pour l'incertitude de la centralisation normative, de la période : les universités, les villes, les parlements, la royauté, les ministres, le pape ne cessent de tenter d'affirmer leur autorité ; et le théâtre profane et polémique ne cesse de témoigner de ces conflits : il y a des textes, mais il y a également beaucoup de données d'archives, de défenses de jouer, de procès contre des auteurs, des acteurs, des imprimeurs même, qui illustrent l'actualité de cette incertitude. Si bien des études historiques ont montré comment Louis XI consolide la nation française, sous Charles VIII, Louis XII et le jeune François $\mathrm{I}^{\mathrm{er}}$, rien ne semble tout à fait clair à cet égard et l'affaire de la décime de 1494 le montre bien. C'est que, à Caen, il s'ensuit une période extrêmement troublante, où les évêques de Caen, de Lisieux, de Coutances s'excommunient mutuellement (les uns ayant la fonction de conserver les privilèges, les autres restant fidèles au roi, ou au pape); où Pierre de Lesnauderie, le scriba convervatoris privilegiorum, descend même, l'épée nue à la main, dans la rue afin de protéger un messager avec un document clé défendant la position de l'université contre le roi (et le pape). Ce n'est pas tout, car les suppôts de l'université réagissent également avec vivacité. On écrit des poèmes, latins et français, et un quidam va même jusqu'à

${ }^{24}$ T. Bonnin, La Farce de Pates-Ouaintes, Evreux, Mancel, 1843. 
afficher (affixit) sur les portes de la cathédrale Saint-Pierre des poèmes latins, en fait des parodies de textes bucoliques, de pastorales à l'antique ( $\mathrm{O}$ Corydon, o Corydon... »), où les images de l'agneau immolé, de la brebis mangée par les loups, de la mère mangée par ses enfants sont déjà largement exploitées. Les textes, en français et en latin (répétons-le), exploitent l'imagerie pastorale à l'antique et ce à un moment où, selon les histoires littéraires traditionnelles (et à ma connaissance elles le sont toutes, sous ce rapport), une telle imagerie ne devrait pas encore être à l'ordre du jour. Qui pis est, non seulement cette poésie publique (ou «publiée ») présuppose une connaissance de ce qui est censé ne pas être connu, mais elle va plus loin au sens où elle paraît même détourner à des fins polémiques, refaçonner à des fins politiques cette poéterie bucolique, la supposant de la sorte connue du public. Il y a donc jeu, il y a donc, me semble-t-il, parodie au plein sens du terme. L'espace public est investi d'une retombée littéraire du conflit, ou faudrait-il dire que le conflit commence à fonctionner au niveau littéraire? C'est ce que nous ne saurons sans doute jamais, le Matrologe de l'Université de Caen restant notre seul document. Il est pourtant tentant de voir, dans les poèmes affichés aux portes de Saint-Pierre, non pas une action littéraire au sujet d'un conflit politique, mais plutôt un événement littéraire (ou que nous qualifierions de «littéraire) qui fait pleinement partie de la polémique en question. Et c'est en ce sens que cet article se veut l'avocat d'une remise en situation, d'une valorisation du caractère éminemment situationnel de la parodie de la fin du Moyen Âge. Le conflit joue dans la rue (et les textes sont dans la rue); le conflit se fait littérature et la littérature commence à agir dans le conflit. Et c'est précisément à ce moment qu'a lieu une représentation théâtrale, encore que celle-ci soit largement codifiée par les circonstances. Car c'est au moment du Mardi Gras que l'inversion commence à reprendre ses pleins droits : c'est l'inversion, la folle parodie. Les étudiants se rendent, au nombre de quelques milliers, armés de torches et d'épées, devant la maison du percepteur de la décime Bureau pour y jouer ce que le manuscrit appelle la farce de Pates Ouaintes. Cette farce rentre donc dans un cadre préconçu de parodies pastorales et n'aurait aucun sens sans le contexte des troubles autour des privilèges universitaires. Au lieu de se définir par rapport à un intertexte, elle se définit à partir d'un moment précis dans un processus de communication. Elle ne se définit pas à partir d'une opposition, mais elle suit d'autres phénomènes historiques et littéraires qui déterminent sa place dans toute une logique de contestation. En d'autres mots : ce qui prime dans le fonctionnement de la parodie, c'est la situation concrète qui a occasionné cette farce et non point la présence d'indices textuels. Le cadre pastoral a été préparé par les poèmes affichés aux portes de la cathédrale Saint-Pierre, le cadre parodique est celui du Mardi Gras, en l'occurrence le moment où la contestation poétique et pamphlétaire peut, apparemment, revêtir un caractère farcesque. En d'autres mots, en ce cas, la parodie est clairement et totalement circonstancielle. Si donc le caractère parodique de cette farce, et le cadre circonstanciel de la parodie dans l'occurrence, semblent bien acquis, le caractère dramatique (à un niveau théorique) l'est bien moins. Ne pourraiton dire que le cortège intégral des suppôts de l'université constitue, en quelque sorte, les acteurs et que le seul percepteur de la décime Bureau (mais regarde-t-il ou se cache-t-il derrière un rideau ?) constitue à lui seul le public? Ne faudrait-il pas, pour les besoins de la cause, revoir nos définitions du théâtre afin d'accorder une place bien plus large au type de théâtre agit-prop, ou drame pragmatique, auquel la 
communauté caennaise a bien pu participer ou assister au $\mathrm{XV}^{\mathrm{e}}$ siècle finissant? De toute manière, la séparation entre le public et les joueurs semble faire problème et le jeu théâtral, ici, ne parle pas tant d'un conflit qu'il n'en fait pleinement partie. En même temps, le caractère «littéraire» de la pièce est clair, qui élabore les images pastorales déjà présentes dans les poèmes polémiques affichés aux portes de la cathédrale, et même (dira-t-on dans une perspective esthétisante) combinant maladroitement deux images traditionnelles qui se laissent mal combiner - en quoi l'Université serait-elle l'agneau aussi bien que la mère, en quoi les adversaires seraient-ils à la fois les loups et les enfants, et même (en poussant les choses légèrement plus loin), en quoi la mère Université serait-elle la mère, la Mère-Église du Christ, menacée par des adversaires comme le Pape de Rome ${ }^{25}$ ? S'il faut résumer ce que le cas de Pates-Ouaintes nous a appris, trois aspects méritent l'intérêt. Sur le plan littéraire, même si les deux images conflictuelles ne révèlent pas une unité de conception, on dira qu'il est question de parodie, de jeux intertextuels, de détournements de formes et de thèmes à d'autres fins. Sur le plan événementiel, par contre, on constate d'abord que, le Mardi Gras étant bien la date par excellence pour des jeux d'inversion et de dérision, le texte ne fait que remplir sa fonction naturelle, ne pourrait être autre que joyeux et ludique et, par là, n'a rien de parodique au sens strict du terme. En troisième lieu, sur le plan de la continuité de l'affaire, on dira que l'inversion que manifeste cette pièce est toute relative car le détournement des images était bel et bien annoncé dans les poèmes affichés aux portes de la cathédrale, donc bien avant le prétexte calendaire du Mardi Gras. Si cette affaire paraît bien mériter une monographie spécialisée comme celle que Le Roy Ladurie a consacrée au Carnaval de Romans en 1580, il faut essayer d'élargir quelque peu la leçon. Le premier point de l'inscription situationnelle étant acquis, penchons-nous sur un autre cas caennais qui implique également Pierre de Lesnauderie.

Quatre ans plus tard, l'Université de Caen joue une autre pièce, également parvenue jusqu'à nous. À Caen, on joue en 1496 La Cène des Dieux, également due à Pierre de Lesnauderie. C'est une pièce curieuse au sens où elle met en scène les dieux de l'Olympe à un moment où, selon toute histoire littéraire, une telle mise en scène n'est pas encore à l'ordre du jour. Que se passe-t-il dans cette pièce ? À l'Olympe, les dieux se réunissent pour décider du sort du genre humain, voilà la situation de départ. Il s'agit d'une occurrence précoce, et non prévue dans l'historiographie générale de la Renaissance de l'Antiquité, de la fonction scénique des dieux antiques. L'éditrice, Eugénie Droz, est même allée jusqu'à supposer que nous ayons ici un exemple unique de théâtre de collège, mais l'on se demande bien d'où elle tire tout cela. Toujours est-il que les dieux débattent la question, décident d'envoyer une maladie mortelle sur la terre. Cette pièce a une certaine unicité : estce une moralité, une farce, une sottie ? En fait, c'est là une question qui n'a qu'une importance toute relative : l'essentiel, croyons-nous, est ailleurs. C'est que ce qui est manifeste, c'est que la Cène reprend la tradition du Procès de Paradis telle qu'on la trouve dans les Mystères de la Passion, où le bon Dieu discute amplement, avec ses

\footnotetext{
${ }^{25}$ Afin de bien comprendre un tel jeu d'oppositions, il faudrait (et c'est urgent) faire un inventaire de telles images, parodiques et situationnelles, au moment de la grande crise gallicane et revoir bien des idées reçues au sujet de la mère Église par rapport à Rome. Si j’y renonce pour le moment, il faut espérer que ce n'est que partie remise.
} 
filles - les émanations divines Paix, Justice, Vérité et Miséricorde - la question du salut des hommes. C'est bien cette question qui est reprise ici par les dieux antiques qui, eux, décident qu'au lieu d'être sauvé, le genre humain doit bel et bien périr. A priori donc, il est question d'un intertexte, voire d'une inversion. S'agit-il aussi d'une parodie? Une analyse littéraire poussée pourra faire apparaître bien des subtilités dans le texte, mais ici encore, ce sont le contexte, la situation et la mise en opposition qui déterminent bien le sens de la pièce. La conjonction de Jupiter et de Saturne de 1494 (nous passons allègrement des dieux aux astres, comme le public du $\mathrm{XV}^{\mathrm{e}}$ siècle), tenue pour responsable de la vague syphilitique qui déferle sur l'Europe (les marins de Christophe Colomb ne seront tenus responsables que bien plus tard), se présente ici comme anti-texte par rapport aux questions du salut dans les mystères. Ce que cherche à nous transmettre la pièce, c'est bel et bien le message selon lequel une conjonction astrologique a déterminé un danger imminent, en l'occurrence la syphilis. Que les auteurs aient choisi pour ce faire une parodie d'une scène bien connue des mystères a une importance déterminante dans notre façon de voir comment cette image fonctionne à l'intérieur d'une affaire bien précise dans un cadre déterminé. Bien plus que le genre, bien plus que de possibles intertextes, ce qui joue ici, c'est avant tout une situation, en l'occurrence reprise à une scène par trop connue des multiples mystères de la Passion. Il y a perversion, au sens où, normalement, le genre humain arrive à s'en tirer, alors que dans notre pièce, il est condamné. Qu'il y ait là un nouvel accent mis sur le caractère péremptoire dans le siècle - sans doute sérieux - ou une joyeuse mise en perspective de la fameuse scène des mystères, reste malheureusement une affaire d'appréciation moderne (et donc une source d'erreur). Qu'à cela ne tienne: même si les intentions premières des farceurs se laissent difficilement définir, le jeu spéculaire entre notre pièce et les Procès de Paradis des mystères est clair, tout à fait manifeste, et devra par là déterminer notre interprétation de la pièce. Sans cela, elle n'a aucun sens, sans cela, elle deviendrait effectivement, comme le voudrait Eugénie Droz, un «seul exemple», ce qui est une faiblesse historiographique, ce qui est un constat d'échec.

Toutefois, quelques années plus tard, sans doute vers 1516, nous aurons droit, à nouveau, à Caen, à une récidive avec Le Grand voyage et pèlerinage de sainte Caquette $^{26}$. Les temps, toutefois, ont changé: Lesnauderie s'apprête à devenir recteur de l'université; ami de Lefèvre d'Étaples, il sera impliqué dans bien des questions religieuses. En même temps, les circonstances de représentation de cette pièce sont moins claires.

Toujours à partir du même point de départ, l'on pourrait citer un cas survenu à Toulouse, en 1508. La pièce jouée, la moralité dite du Nouveau Monde, constitue une autre instance parodique nette, mais implicite. Ce que nous connaissons, notre source d'information, notre unique source d'information, est un imprimé parisien de 1511, dû à l'imprimeur parisien Guillaume Eustache. Toutefois, cette pièce est associée, dans l'imprimé, à une autre pièce appelée la Sotise à huit personnages, qui a été attribuée à André de la Vigne (et à bien d'autres, mais passons...). La Sotise a une page de titre qui lui appartient, mais la moralité a une page de titre qui promet à la fois l'Estrif... et le Nouveau monde (en fait, c'est une erreur, car cette moralité ne

\footnotetext{
${ }^{26}$ A. Tissier, Recueil de farces (1450-1550), t. II, Genève, 1987, p. 17-72.
} 
devrait pas s'appeler le Nouveau Monde, mais plutôt l'Estrif de.... $)^{27}$. Nous sommes à Toulouse, en 1508. La moralité de l'Estrif... a été jouée à la Pentecôte : c'est la moralité, mais au Mardi Gras (probablement), c'est la Sotise que l'on a jouée. Toutefois, cet Estrif a été joué sur la place Saint-Étienne (à Toulouse, c'est la place de la cathédrale) et le personnage Université date la pièce dans son verdict final. Les choses commencent à prendre leur plein sens lorsque l'on se rend compte que cette date est bien celle de la Pentecôte de 1508 et que, par là, la glorification de la Pragmatique Sanction, dans cette pièce autour des libertés gallicanes, reçoit un nouveau sens. C'est que la Pentecôte, date de l'effusion du Saint Esprit - et par là aussi date par excellence pour jouer des jeux de la Passion - se présente ici comme une occasion rêvée pour défendre la Pragmatique qui, selon le texte même, a été donnée au Concile de Bâle par le Saint Esprit lui-même. Cette mise en situation, cette inscription calendaire, change profondément le sens de la moralité : là où les mystères de la Passion nous montrent comment s'effectue le salut des hommes, malgré l'opposition de Lucifer par le biais de son agent Satan, la moralité nous montre comment la Pragmatique peut être réinstaurée malgré l'opposition du Pape, du roi (le prince Quelqu'un) et de l'Ambitieux. Cette moralité, qui, en elle-même, est bien polémique, voire virulente (contre le roi de France, contre le Pape) acquiert un sens tout à fait différent à cause de son inscription calendaire et, partant, à cause de la manière dont elle thématise, effectivement, les qualités requises de son contexte de représentation.

Disons en guise de conclusion que le contexte de la fête prime. Fêtes d'inversion qui secrètent des textes d'inversion ou polémiques d'inversion qui secrètent des fêtes : c'est la contextualisation, parfois dans un sens assez large, qui détermine le comment et pourquoi du fonctionnement parodique. Dans le contexte général de l'inversion festive, il n'y a peut-être plus lieu de parler de parodie, car il y a une logique interne qui relie le texte à son contexte, en d'autres mots : il n'y a pas de rupture esthétique ou stylistique, mais plutôt une continuité.

Il est possible d'élargir l'idée situationnelle en faisant valoir que ce n'est pas nécessairement le caractère événementiel de telles parodies qui détermine leur fonction et leur bonne compréhension. Ce que les historiens du théâtre médiéval ont trop peu fait, et ce qui a bien, par là, entravé une compréhension plus historique de sa représentation médiévale, c'est l'étude d'un contexte plus large. Élémentaires, en tout cela, sont les études respectives d'Emmanuel Le Roy Ladurie au sujet du Carnaval de Romans en 1580 (qui, lui, étudie amplement ce contexte plus large sans, malheureusement, disposer d'un moment précis, d'un événement représentationnel qui puisse cristalliser ce contexte) et de Natalie Zemon Davis qui, à son tour, définit le contexte d'une façon large - surtout dans la chronologie-, à tel point que le général tue le particulier, que la spécificité temporelle et locale s'estompe devant des interprétations préconçues. Quelques exemples méritent d'être cités.

Ainsi, et c'est un détail certainement négligé par les savants, que les farces et sotties parisiennes apparaissent comme un répertoire, avec des intertextes mutuels non négligeables, avec des renvois internes au répertoire qui présupposent une espèce de bagage culturel de la part du public, qui présupposent également une

${ }^{27}$ Voir O. Duhl, Sotise a huit personnaiges [Le Nouveau Monde], Genève, Droz, 2005. 
participation suivie d'un même public au même type de représentations, devrait déterminer aussi, en toute logique, la place de la parodie. Tel texte parodique qui se définit par rapport à tel autre texte parodique nous transmet une autre idée de la parodie - si seulement on prend la peine de les mettre en rapport. De la parodie à la satire ou: de l'inversion à la subversion. Il s'agit d'un thème problématique et amplement problématisé dans les multiples études sur le Carnaval. Ce que, toutefois, l'article que l'on vient de lire entend bien montrer, c'est que la question de la parodie au théâtre est bien trop importante pour la laisser aux « littéraires », qu'audelà des intertextes, il y a plus : une vie représentationnelle. Ce qui vaut également pour toute littérature à destination performantielle, toute littérature de la voix comme opposée à celle de la lettre.

Jelle Koopmans

Université d'Amsterdam 